\title{
Immunoelectron Microscopic Localization of Late Envelope Proteins of the XP-5 Family in Mouse Epidermis
}

\author{
V.V. Speransky, ${ }^{*}$ Z. Zhou, ${ }^{* *}$ D.R. Roop, ${ }^{* *}$ and A.C. Steven* \\ * Laboratory of Structural Biology, NIAMS/NIH, 50 South Dr. MSC 8025, Bethesda, MD 20892 \\ ** Department of Molecular and Cellular Biology, Baylor College of Medicine, One Baylor Plaza, \\ Houston, TX 77030
}

The cornified cell envelope (CE) is a 15-nm layer of covalently cross-linked protein and lipid that replaces the plasma membrane in terminally differentiating keratinocytes [1] and is thought to play a key role in epidermal barrier function. Its main protein constituent $(70-80 \%)$ is loricrin. The observations that loricrin knockout (LKO) mice show only mild symptoms and have CEs of normal thickness led to the hypothesis that loricrin is substituted by other protein(s) in these animals [2]. A candidate is the recently discovered late envelope proteins (LEPs) of the XP-5 family, which are also expressed in wild-type epidermis [3,4]. LEPs have sequence similarity to loricrin and are upregulated in LKO skin. In the present study, we have used immunogold labeling to test whether the LEPs are indeed components of the CE and to study their localization in the epidermis.

$1 \mathrm{~mm}$ squares of wild type and LKO neonatal mouse skin were fixed and prepared for cryosectioning according to the Tokuyasu technique. Thawed cryosections were labeled with an affinity-purified antibody raised against a 15-residue peptide that is highly conserved in all XP-5 proteins. All layers of the stratum corneum (SC) were LEP-positive. The innermost 2-5 layers labeled densely all over the cell, whereas in the outer layers the gold particles were mostly found along the cell borders (Fig. 1). In the stratum granulosum, label was localized to large irregularly shaped granules, known from previous work as F-(filaggrin)-granules (Fig. 2, [5]). Label was completely absent anywhere below the stratum granulosum, and control sections incubated in parallel with an unrelated antibody showed no labeling. In corneocytes where the labeling was peripheral, many gold particles were found on the outside of the CE. To quantify this impression, we measured distances between the gold particles and border of the cell on sections where the latter was clearly defined, and compared their distribution to that of loricrin, which is known to reside on the inner side of the CE (Fig. 3). The XP-5 proteins displayed a wider spread than loricrin, including a peak outside the cell.

Our data confirm that the XP-5 proteins are indeed associated with the CE, and suggest two modes of association with the CE - one external and one internal. We also found that these proteins' biosynthetic pathway involves F-granules, with subsequent release into the cytoplasm as the cell undergoes cornification. Their pathway differs from that of loricrin and appears similar to, and possibly related with, that of filaggrin [6].

\section{References}

[1] D.R. Roop, Science 267 (1995) 474.

[2] M. Jarnik et al., J. Invest. Dermatol. 118 (2002) 389.

[3] D. Marshall et al., P.N.A.S. 98 (2001) 13031.

[4] Z. Zhou and D.R. Roop, unpublished data.

[5] A.C. Steven et al., J. Struct. Biol. 104 (1990) 150.

[6] We thank Drs D. Winkler and N. Cheng for providing EM facilities. 

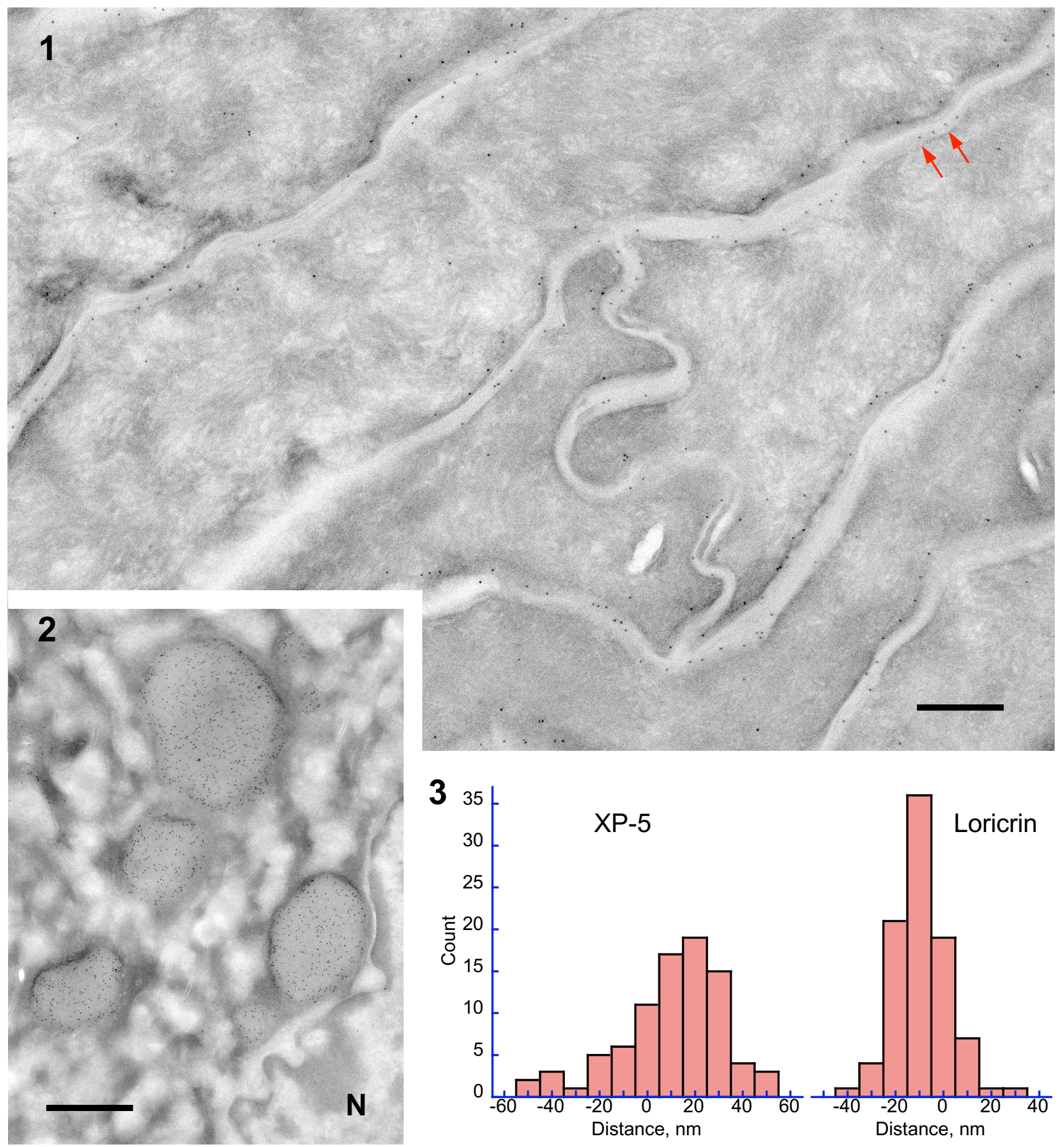

Fig. 1. Stratum corneum of the neonatal mouse epidermis labeled with an antibody against the XP-5 LEP family. Labeling was visualized by Protein A-gold. Note the gold particles outside the CE (arrows). Bar $=0.5 \mu \mathrm{m}$.

Fig. 2 LEP-positive granules in a stratum granulosum cell. $\mathrm{N}$ - nucleus. Bar $=1 \mu \mathrm{m}$.

Fig. 3. Distribution of gold-to-cell border distances measured on sections labeled with the XP-5 family antibody and an antibody to loricrin. Direction to the outside of the CE was arbitrarily given positive sign. 